\title{
Testing for Mycobacterium tuberculosis infection using the QuantiFERON-TB GOLD assay in patients with comorbid conditions in a tertiary care endemic setting
}

\author{
Kiran Iqbal Masood ${ }^{1}$, Bushra Jamil ${ }^{2}$, Alnoor Akber ${ }^{1}$, Maheen Hassan ${ }^{1}$, Muniba Islam ${ }^{1}$ and Zahra Hasan ${ }^{1 *}$ (D)
}

\begin{abstract}
Introduction: There were 10 million new cases of tuberculosis (TB) in 2017. To eliminate TB, it is necessary to diagnose active TB and latent tuberculosis infection (LTBI). Diagnosis of paucibacillary disease and in extrapulmonary TB (EPTB) remains challenging; low mycobacterial load can be missed by microbiological or molecular based confirmation; EPTB, can be misdiagnosed due to absence of site specific specimens for testing. Interferon gamma release assays (IGRA) use T cell-based Interferon-gamma (IFN- $\gamma$ ) to identify infection with $M$. tuberculosis (MTB) but cannot discriminate between active and LTBI. We investigated how IGRA was being used in a high burden low resource setting.

Methods: We conducted a retrospective review of 149 consecutive cases received for QuantiFERON-TB Gold InTube Assay (QFT-GIT) testing in routine clinical service.

Results: Fifty-six cases were QFT-GIT positive and 93 were QFT-GIT negative. Thirty-six per cent of QFT-GIT tested cases had active TB. Of QFT-GIT positive cases, 59\% patients had active TB; 10 with pulmonary and 23 with extrapulmonary TB. The remaining 41\% QFT-positive cases were LTBI. Of the QFT-GIT negative cases, 22\% had active TB. Co-morbid conditions were present in 37\% of QFT-GIT positive and 60\% of QFT-GIT negative cases.

Conclusions: Our study shows that IGRA is being used as an adjunct test for active TB in this population. It highlights the complexity of interpreting QFT-GIT results particularly for QFT-GIT negative cases when ruling out MTB infection.

Keywords: Tuberculosis, QuantiFERON-TB GOLD, Co-morbids, Interferon gamma release assay, Pulmonary TB, Extrapulmonary TB, Latent TB, Active TB, Endemic, Immunocompromised
\end{abstract}

\section{Introduction}

Globally, an estimated 10 million new cases of tuberculosis (TB) were reported in 2017 [1]. Pakistan ranks 5th amongst high TB burden countries with 525,000 new cases reported [1]. It is necessary to correctly diagnose both active TB and latent tuberculosis infection (LTBI) in order to achieve the End TB target by 2035 as proposed by the World Health Organization and reduce TB incidence by $95 \%$ as compared with 2015 [2,3].

\footnotetext{
* Correspondence: zahra.hasan@aku.edu

'Department of Pathology and Laboratory Medicine, The Aga Khan

University, Stadium Road, P.O.Box 3500, Karachi, Pakistan

Full list of author information is available at the end of the article
}

Pulmonary disease is the predominant form of tuberculosis, although, extra pulmonary tuberculosis (EPTB) including skeletal, abdominal, genito-urinary, lymph nodes, kidney, pleural and meninges remains common [4]. Accurate diagnosis followed by appropriate management of TB is essential for controlling disease transmission. Diagnosis of pulmonary and EPTB primarily depends on a positive radiology result and microbiological confirmation through microscopy, mycobacterial culture [4] and/ or TB Xpert MTB/RIF assay (Cepheid, USA) testing. However, TB poses diagnostic dilemmas especially with regards to EPTB cases where it may not be possible to obtain an appropriate site

(c) The Author(s). 2020 Open Access This article is distributed under the terms of the Creative Commons Attribution 4.0 International License (http://creativecommons.org/licenses/by/4.0/), which permits unrestricted use, distribution, and 
specific specimen for laboratory confirmation or, it may be technically complex or financially unfeasible to do so.

For latent $\mathrm{TB}$, the tuberculin skin test (TST) employing purified protein derivative antigen (PPD) is used most frequently [5]. Interferon gamma release assays (IGRAs) such as, QuantiFERON TB Gold in tube assay (QFT-GIT) measure specific $\mathrm{T}$ cell-based responses to MTB-specific antigens including Early secretory antigen target (ESAT)-6, Culture filtrate protein (CFP)-10 and TB 7.7 [6]. T SPOT-TB is an IGRA which requires purified mononuclear cells to quantify IFNgamma (IFN- $\gamma$ ) responses [7]. QFT- GIT uses whole blood and is more convenient to use but may be affected by the number of mononuclear cells present in the test sample [7]. QFT-GIT is more specific than TST for identification of LTBI, is unaffected by BCG vaccination [8] and can be useful as a confirmatory test to rule out false positives results [9].

A consideration in the interpretation of IGRA based results is the competence of individual immune responses. As QFT-GIT is reliant on a robust $\mathrm{T}$ cell response, results in young children, severely malnourished subjects or those who are HIV infected, may be compromised [10]. Immunosuppression and lymphocytopenia tend to affect results of QFT-GIT and lead to indeterminate results [11].

QFT-GIT testing has been used in differential diagnosis of active TB in multiple studies [12]. There are variable data regarding the association between QFT-GIT positive results and active TB [12]. The overall sensitivity for QFT-GIT to diagnose active tuberculosis in a metaanalysis conducted by Metcalk J et al. was found to be 69-83\% [13]. Another meta-analysis conducted by Dai Y et al. [14] reported the overall sensitivity of QFT-GIT for TB diagnosis to be $85 \%$ and specificity to be $84 \%$. Usage of QFT-GIT has been recommended as a rule out active tuberculosis in areas with low prevalence of tuberculosis $[15,16]$. Due to its higher cost as compared with the TST it is not routinely recommended for screening of LTBI in high burden low middle income countries (LMIC) [17]. However, TST is recommended for screening at risk groups such as patients prior to initiation of anti-tumour necrosis factor (TNF) treatment, patients receiving dialysis, or patients preparing for organ or haematologic transplantation [5].

IGRA testing using the QFT-GIT assay was initiated at the Clinical Laboratory, The Aga Khan University Hospital (AKUH), Pakistan in 2013. We conducted this retrospective review to understand the cohort of patients in whom IGRA testing was being requested. There is currently no published data regarding the performance of IGRA testing in this population. This study reveals important insights into the context in which IGRA testing is routinely being performed and gives relevance to its utility as an adjunct test for EPTB.

\section{Methods}

\section{Study subjects}

This was a retrospective analysis of cases for whom QuantiFERON-TB Gold In Tube assay (QFT-GIT; Cellestis, Ltd. Germany) was performed at AKUH Clinical Laboratory, Karachi, Pakistan during the period July to December 2013. One hundred and forty-nine hospital in-patient cases for whom QFTGIT tests were referred by physicians as per their routine laboratory work were included in the study in a consecutive sampling method. Laboratory data and hospital medical records were reviewed, demographic data was retrieved, clinical history of the patient and comorbid conditions were documented and assessed for correlation with QFT-GIT results.

\section{QuantiFERON-TB gold in-tube assay}

One milliliter of whole blood from each study subject was added to each of the three tubes; TB antigen (ESAT-6, CFP-10 and TB 7.7), mitogen (positive control) and Nil (negative control) provided with QFTGIT and processed as per manufacturer's instructions. The tubes were incubated for 16 to $24 \mathrm{~h}$, plasma was harvested and IFN- $\gamma$ concentrations $(\mathrm{IU} / \mathrm{mL})$ in plasma was measured by an ELISA reader at $450 \mathrm{~nm}$ and calculated by the 'QFT-TB-analysis Software'. A cut-off of IFN- $\gamma \geq 0.35 \mathrm{IU} / \mathrm{ml}$ was used. A determinate test must have mitogen minus negative control $\geq 0.5$ $\mathrm{IU} / \mathrm{ml}$ and/or $\mathrm{TB}$ antigens minus negative control $\geq 0.35 \mathrm{IU} / \mathrm{ml}$.

\section{Classification of study subjects}

Cases were categorized based on a review of hospital clinical and laboratory records for each patient. Cases were grouped into those with 'Active TB', 'LTBI' and 'Non-TB'. Cases with 'Active TB' were defined as cases positive for MTB culture/ acid-fast bacilli smear/ histopathology predictive of granulomatous inflammation had a strongly suggestive chest X-ray with matching clinical history and/ or responded to antituberculous therapy (ATT). Patients had both 'PTB', and 'EPTB'. ЕPTB included sites; lymph node (LN), abdominal, tuberculous meningitis (TBM), central nervous system (CNS), pelvic, pleural, peritoneal, thyroiditis, knee and spine.

LTBI were QFT-GIT positive cases with a negative diagnosis of active TB (as defined by above mentioned criteria). Non-TB cases were QFT-GIT negative with a negative diagnosis of active $\mathrm{TB}$ (as defined by above mentioned criteria).

\section{Statistical analysis}

Statistical analysis was carried out using the Graphpad PRISM and Statistical Packages for Social Sciences 
(SPSS). Data was presented as median values. The Chi squared test was used to compare different parameters between QFT-GIT positive and negative groups.

\section{Results}

\section{Characteristics of study subjects}

Of the 149 cases we reviewed, 56 (37.5\%) had a positive QFT-GIT result and $93(62.5 \%)$ had a negative QFTGIT result. The results of all cases are illustrated in Fig. 1. No indeterminate results were obtained. Age and gender were found to be comparable between the QFTGIT positive and negative groups (Table 1). Nineteen cases (34\%) in the QFT-GIT positive group and 37 (40\%) cases in QFT-GIT negative group were observed to have lymphopenia. Further, we evaluated the cases for co-morbid conditions and identified a range of conditions such as auto-immune disease (including, Rheumatoid arthritis, Systemic Lupus Erythematosis and mixed connective tissue disorders), chronic kidney disease, malignancy, chronic liver disease, chronic obstructive pulmonary disease, achalasia, chronic heart disease, epilepsy and endocrine disorders. Of the 56 QFT-GIT positive subjects, $35(63 \%)$ did not have any co-morbid conditions whilst 21 (27\%) did so. Of the 93 QFT-GIT negative subjects, 37 (40\%) did not have comorbid conditions whilst $56(60 \%)$ had known co-morbid conditions. There were a significantly greater $(p=0.007)$ number of individuals with co-morbid conditions in the QFT-GIT negative group

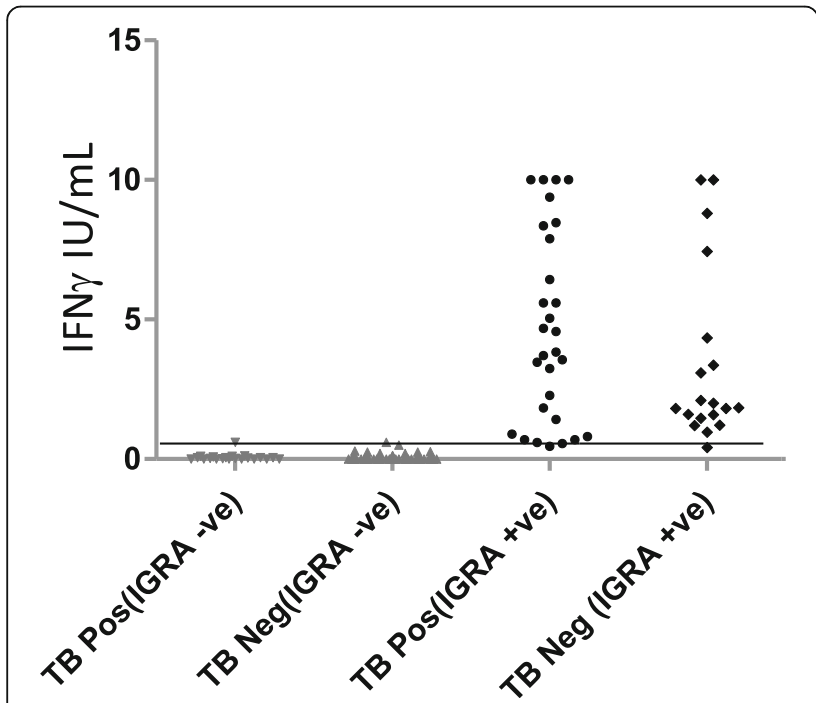

Fig. 1 IFNy titers in QFT-GIT negative and QFT-GIT positive TB patients. Whole blood from study subjects was incubated in QFT-GIT (NIL, TB and mitogen) tubes for $18 \mathrm{~h}$. ELISA was performed to detect IFN- $\gamma$ levels. Data is represented in the form of scatter plots. Horizontal line indicates cut-off at $0.35 \mathrm{lU} / \mathrm{mL}$
More individuals in the QFT-GIT negative group had autoimmune disease $(p<0.001)$.

\section{Latent and active TB in QFT-GIT positive cases}

Patients with active TB were identified in both QFTGIT positive and negative cases (Table 2). Of the 56 QFT-GIT positive cases, 33 were found to have active disease and 23 were identified as LTBI. The IFN $\gamma$ levels from QFT-GIT testing is illustrated in Fig. 1. The range of positive values was comparable between LTBI, PTB and EPTB groups (data not shown).

Of the QFT-GIT positive active TB cases, these included ten PTB and twenty-three EPTB cases. The EPTB cases had TB at different sites including, lymph nodes, abdominal, TB meningitis and tuberculomas, pelvic, pleural, peritoneal, thyroid, knee and the spine (Table 3).

\section{Active TB in QFT-GIT positive cases}

Of the ninety-three QFT-GIT negative cases reviewed, $20(21.5 \%)$ patients were found to have active TB; 3 with pulmonary and 17 with EPTB (Table 3). Follow up clinical records showed that these patients with $\mathrm{TB}$ were subsequently put on anti-tuberculous therapy (ATT) and all of them showed a positive response to treatment as assessed by clinical and radiological improvement at the end of treatment completion.

Seventy-three QFT-GIT negative cases who did not have signs and symptoms of active disease were categorized as being unlikely of having MTB infection, as nonTB cases.

Further, a sub-group analysis was conducted for using QFT-GIT to identify active TB in our study cohort. For this, the TB positive (IGRA negative) were taken as False Negatives $(n=20)$, TB negative (IGRA negative) as True Negatives $(n=93)$, TB Positive (IGRA positive) as True Positives $(n=33)$ and TB Negative (IGRA positive) as False Positives $(n=23)$. This sub-group analysis gave a sensitivity of $62.3 \%$ and a specificity of $76 \%$ for IGRA based identification of active TB infection in our cohort.

\section{Hematological characteristics and comorbid conditions} Hematological characteristics and the IFN- $\gamma$ responses of QFT-GIT positive and negative groups were compared. Patients in the QFT-GIT group had a range of co-morbid conditions such as, chronic kidney disease, diabetes mellitus, malignancy, epilepsy and patients with more than one chronic illness $(n=21,37.5 \%)$, Table 4 . The TB antigen and mitogen results of the QFT-GIT test and lymphocyte counts for the cases sub-divided into different co-morbid conditions are illustrated in Table 4. Patients with malignancy had higher lymphocyte counts than the normal range $\left(<1500\right.$ cells $\left./ \mathrm{mm}^{3}\right)$ while patients with type 2 diabetes had lower lymphocyte counts 
Table 1 Baseline characteristics of study subjects

\begin{tabular}{|c|c|c|c|c|}
\hline & Overall (n) & QFT positive (n) & QFT negative (n) & $P$ value \\
\hline Age & 149 & 56 & 93 & NS \\
\hline Sex (female) & 77 & 28 & 49 & 0.75 \\
\hline Sex (male) & 72 & 28 & 44 & 0.75 \\
\hline Lymphopenia* & 56 & 19 & 37 & 0.665 \\
\hline Autoimmune disease & 7 & 0 & 7 & $<0.001$ \\
\hline Chronic kidney disease & 7 & 2 & 5 & 0.613 \\
\hline Renal transplant & 2 & 0 & 2 & 0.331 \\
\hline Malignancy & 15 & 4 & 11 & 0.357 \\
\hline Chronic liver disease & 2 & 0 & 2 & 0.331 \\
\hline COPD & 1 & 0 & 1 & 0.439 \\
\hline Achlasia & 1 & 1 & 0 & 0.439 \\
\hline Coronary heart disease & 1 & 0 & 1 & 0.439 \\
\hline Diabetes & 20 & 7 & 13 & 0.809 \\
\hline Other endocrine disorders & 1 & 0 & 1 & 0.439 \\
\hline Epilepsy & 2 & 1 & 1 & 0.744 \\
\hline Multiple co-morbids & 18 & 6 & 12 & 0.096 \\
\hline No Known comorbids & 72 & 35 & 37 & 0.007 \\
\hline
\end{tabular}

'*' lymphopenia defined as lymphocytes $<1500$ cells/mm3; 'QFT', Quantiferon-Gold in Tube assay (Cellestis, Germany); Cut-off for QFT positive $\geq 0.35$ IU/ml.**' lymphopenia is defined as lymphocytes $<1500$ cell $\mathrm{s} / \mathrm{mm} 3$; ' 't' ' patients with multiple diseases including, diabetes, chronic liver disease, chronic kidney disease, endocrine disorders (panhypo-pitutiarism, hypothyroidism, thalassemia and malignancy); ${ }^{* * *}$ autoimmune disease includes Rheumatoid arthritis, Systemic Lupus Erythematosis and mixed connective tissue disorders. COPD, chronic obstructive pulmonary disorder. QFT negative and QFT positive groups are compared using Chi-square test. $P$ value $\leq 0.05$ is considered to be significant between the two groups.

than normal levels. Overall, 35 (62.5\%) did not have any known co-morbid conditions.

Evaluation of QFT-GIT negative cases revealed that $39 \%$ (36 out of 93) did not have any comorbid conditions whilst $61 \%$ (57 out of 93 ) had a range of conditions as identified in Table 4. QFT-GIT IFN- $\gamma$ responses and lymphocyte counts were compared for QFT-GIT negative cases with and without comorbid conditions for sub-groups of Active TB and Non-TB. Of the 20 cases with active TB, $8(40 \%)$ of cases had comorbid conditions whilst $12(60 \%)$ did not.

Table 2 Diagnostic details for patients with active TB

\begin{tabular}{cccccccc}
\hline & N & AFBC & Microscopy & Radiology & ATT & Histo & PCR \\
\hline QFT +ve & & & & & & & \\
PTB & 10 & 4 & 0 & 10 & 8 & 2 & N/A - \\
EPTB & 23 & 6 & 0 & 11 & 14 & 5 & 1 \\
QFT -ve & & & & & & & \\
PTB & 3 & 1 & 1 & 2 & 3 & 0 & 1 \\
EPTB & 17 & 0 & 0 & 5 & 9 & 7 & N/A
\end{tabular}

QFT QuantiFERON-Gold In Tube Assay, PTB Pulmonary TB, EPTB Extrapulmonary TB, $N$ Number of subjects, AFBC Acid fast bacilli culture by MIGIT system (Becton Dickinson, USA); Microscopy, acid fast bacillus smear using Ziehl Neelsen staining; Radiology, XRay/ CT scan/ or MRI; ATT, anti-tuberculosis treatment response; histo-pathological staining of biopsy material and or FNAC (fine needle aspirate cytology) where relevant. The numbers indicate those diagnosed as positive by each method. N/A, not available
Sixty-seven percent (49 out of 73) of the QFT-GIT negative non-TB group had comorbid conditions. Of these, patients with diabetes had the lowest lymphocyte counts (Table 5).

\section{Discussion}

The Aga Khan University Hospital Karachi, Pakistan is a tertiary care referral center for the region. The AKUH receives a large proportion of cases requiring complex clinical and surgical care. We conducted this retrospective study of samples received for routine QFT-GIT testing at the AKUH Clinical laboratory to understand how the IGRA test was performing in the cohort of cases received. This is the first such data on the utility of IGRA testing in Pakistan.

Robust identification of MTB infection is important for both latent and active disease. In endemic populations, LTBI particularly in high risk populations need to be identified and treated appropriately [17]. Key recommendations of the WHO Guidelines on management of latent tuberculosis identify groups in whom LTBI testing is strongly recommended. These include, people living with HIV, contacts of pulmonary TB cases, patients initiating anti-tumor necrosis factor therapy, patients receiving dialysis and patients preparing for transplantation [5]. Individuals with suppressed immune response are at 
Table 3 Characteristics of study subjects

\begin{tabular}{|c|c|c|c|c|c|}
\hline TB Status & $\begin{array}{l}\text { Total } \\
(n=149)\end{array}$ & $\begin{array}{l}\text { Male to female ratio } \\
M ; F\end{array}$ & $\begin{array}{l}\text { Age, years } \\
\text { (Median) }\end{array}$ & $\begin{array}{l}\text { TLC } \\
\left(10 e^{3} / L\right)\end{array}$ & Lymphocyte count (\%) \\
\hline \multicolumn{6}{|c|}{ QFT Positive $(n=56)$} \\
\hline Active TB & $(n=33)$ & & & & \\
\hline PTB & 10 & $3: 7$ & 44 & 7.9 & 17.25 \\
\hline EPTB & 23 & $8: 15$ & 44 & 7.85 & 24.1 \\
\hline Latent TB & 23 & $17: 6$ & 50 & 8.5 & 22.05 \\
\hline QFT Negative & $(n=93)$ & & & & \\
\hline Active TB & $(n=20)$ & & & & \\
\hline PTB & 3 & $0: 3$ & 18 & 7 & 27.8 \\
\hline EPTB & 17 & $9: 8$ & 45 & 9.4 & 17.2 \\
\hline Non TB & 73 & $35: 38$ & 54 & 8.5 & 21.3 \\
\hline
\end{tabular}

'TLC', total lymphocytes count; 'QFT', Quantiferon-Gold in Tube assay (Cellestis, Germany); 'PTB', pulmonary TB; 'EPTB', extrapulmonary TB (lymph node, abdominal, TB meningitis, tuberculoma, pelvic, pleural, thyroid, knee and spine). 'M: $F^{\prime}$, male: female; 'Active TB', subjects with a confirmed TB diagnosis based on positive radiology, microbiology, microscopy testing and/or a positive treatment response; 'Probable 'Latent TB' cases were QFT-G positive without a confirmed diagnosis of TB; 'Non-TB', cases were QFT-G negative and without a confirmed diagnosis of TB. Cut-off for QFT positive $\geq 0.35$ IU/ml. Lymphopenia is defined as lymphocytes $<1500$ cells $/ \mathrm{mm} 3$. Data is presented as Median values

an increased risk of developing active tuberculosis as compared to the general population $[18,19]$.

The QFT-GIT is a specific and convenient tool for screening MTB infection [20]. The WHO does not recommend the replacement of TST with this technology within low- and middle-income countries as it would be more costly [5]. However, QFT-GIT is more specific than TST for identification of MTB infection and therefore can reduce overestimation of infection and treatment rates [21, 22]. In 2017, QFT-GIT was replaced by the QFT Plus (Qiagen) which has two different TB antigen tubes for $\mathrm{T}$ cell recognition allowing further improvements in MTB specific antigen detection [23].

We found that $59 \%$ of the QFT-GIT positive cases we studied had active TB. In all, $18 \%$ of QFT-GIT cases had PTB whilst $41 \%$ had EPTB. QFT-GIT positive results in active TB are in line with previous studies which show IGRAs to have high specificity in detection of individuals with exposure to MTB [12]. For active TB case finding it is recommended that clinical signs and symptoms of TB be documented prior to testing for LTBI in addition to chest radiography. The remaining $41 \%$ of QFT-GIT positive cases were post probably LTBI where testing had been performed for various reasons such as, screening post-exposure to TB patients and unexplained febrile illness.

We investigated the QFT-GIT negative cases and found that 20 of them (22\%) had Active TB. Most of these (17 of 20) had EPTB. Therefore, it appeared that here, QFT-GIT testing had been performed as an adjunct test for active TB case finding. Of the QFT-GIT negative cases, 9 were on steroid treatment, one had autoimmune disease and one had received chemotherapy. Patients with malignancy among QFT-GIT negative subjects with active TB patients had lowered lymphocyte counts than the normal range. As QFT-GIT relies on effective $\mathrm{T}$ cell responses, the interpretation of the test needs to take into account factors that influence host immune parameters. There is not much data available on the interpretation of QFT-GIT in patients with

Table 4 Co-morbid conditions present in QFT positive study subjects

\begin{tabular}{|c|c|c|c|c|c|}
\hline Co-Morbid & N (\%) & $\begin{array}{l}\text { TB Ag } \\
\text { (IFNy IU/ml, Median) }\end{array}$ & $\begin{array}{l}\text { Mitogen } \\
\text { (IFNy IU/ml, Median) }\end{array}$ & $\begin{array}{l}\text { TLC } \\
(10 \text { e3/L) } \\
\end{array}$ & Lympho (\%) \\
\hline No Known comorbids & $35(62.5)$ & 4.68 & 10 & 7.8 & 22.4 \\
\hline Diabetes & $7(12.5)$ & 1.82 & 10 & 9.3 & $15.9^{\#}$ \\
\hline Malignancy & $4(7)$ & 1.975 & 2.96 & $11.3^{*}$ & 44.35 \\
\hline Achlasis & $1(1.8)$ & 0.59 & 2.47 & 6.2 & 38 \\
\hline Chronic Kidney disease & $2(3.6)$ & 5.91 & 4.795 & 8.8 & 23.3 \\
\hline Epilepsy & $1(1.8)$ & 1.59 & 10 & 7 & 23.4 \\
\hline Multiple comorbids & $6(10.7)$ & 1.51 & 5.78 & 8.2 & 20.5 \\
\hline
\end{tabular}

'TLC', total lymphocytes count; 'QFT', Quantiferon-Gold in Tube assay (Cellestis, Germany). QFT results for TB antigen (TB Ag) and Mitogen are provided. Cut-off for QFT positive $\geq 0.35 \mathrm{IU} / \mathrm{ml}$. “* Denotes values greater than the normal range; '\#' Denotes values less than the normal range. Lymphopenia defined as lymphocytes $<1500$ cells/mm3. '\#' patients with multiple co-morbids including, diabetes, chronic liver disease, chronic kidney disease, endocrine disorders (panhypopitutiarism, hypothyroidism, thalassemia and malignancy) 
Table 5 Co-morbid conditions present in QFT negative study subjects

\begin{tabular}{|c|c|c|c|c|c|}
\hline \multicolumn{6}{|c|}{ Subjects with Active TB $(n=20)$} \\
\hline Co-morbids & Frequency $(n, \%)$ & TB Ag (IFNy IU/ml, Median) & Mitogen (IFNy IU/ml, Median) & TLC (\%) & Lymphocytes (\%) \\
\hline None known & $12(60)$ & 0.025 & 10 & 8.1 & 18.2 \\
\hline Diabetes & $3(15)$ & 0.07 & 5.79 & 8.1 & 19.4 \\
\hline Other Endo & $1(5)$ & 0.03 & 8.27 & 13.2 & 19 \\
\hline Auto immune disease $\mathrm{e}^{\mathrm{a}}$ & $1(5)$ & 0.01 & 1.85 & 10.7 & 15.9 \\
\hline Malignancy & $1(5)$ & 0.04 & 0.77 & 109 & 1 \\
\hline $\mathrm{CHD}$ & $1(5)$ & 0.01 & 10 & 7 & 32 \\
\hline Multiple Co-morbids ${ }^{\mathrm{b}}$ & $1(5)$ & 0.1 & 4.08 & 7.7 & 17.7 \\
\hline \multicolumn{6}{|c|}{ Subjects with Non-TB $(n=73)$} \\
\hline Co-morbids & $\begin{array}{l}\text { Frequency } \\
(n, \%)\end{array}$ & $\begin{array}{l}\text { TB Ag } \\
\text { (IFNy IU/ml, Median) }\end{array}$ & $\begin{array}{l}\text { Mitogen } \\
\text { (IFNy IU/ml, Median) }\end{array}$ & TLC (\%) & $\begin{array}{l}\text { Lymphocytes } \\
(\%)\end{array}$ \\
\hline None known & $24(33)$ & 0.025 & 10 & 7.4 & 23.6 \\
\hline Chronic Kidney Disease & $7(10)$ & 0.04 & 8.15 & 7.8 & 20.6 \\
\hline Chronic Lung Disease & $3(4)$ & 0.04 & 7.66 & 5.8 & 17.9 \\
\hline Diabetes & $10(14)$ & 0 & 7.81 & 11 & 11.8 \\
\hline Auto Immune disease ${ }^{a}$ & $6(8)$ & 0.01 & 10 & 9.4 & 17 \\
\hline Malignancy & $10(14)$ & 0.065 & 6.88 & 8.1 & 23.5 \\
\hline Epilepsy & $1(1)$ & 0.03 & 10 & 5.8 & 38.4 \\
\hline Coronary Heart Disease & $1(1)$ & 0 & 4.29 & 24.4 & 12 \\
\hline Multiple co-morbids & $11(15)$ & 0 & 10 & 36 & 21.3 \\
\hline
\end{tabular}

'TLC', Total lymphocyte count; 'QFT', Quantiferon TB Gold in tube assay; 'COPD', Chronic Obstructive Pulmonary Disorder. Cut-off for QFT positive is $\geq 0.35$ IU/ml.

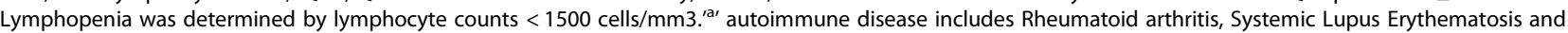
mixed connective tissue disorders. ' ${ }^{\text {' }}$ patients with multiple diseases including, diabetes, chronic liver disease, chronic kidney disease, endocrine disorders (panhypo-pitutiarism, hypothyroidism, thalassemia and malignancy)

significant underlying illnesses. We found lymphopenia and lowered IFN- $\gamma$ values to be associated with conditions such as, chronic kidney disease, renal transplant patients, diabetes, autoimmune disease, chronic liver disease, COPD, malignancy, epilepsy, chronic heart disease, multiple co-morbidities and others with no known major coexisting medical conditions. Previous studies have shown that therapeutic levels of dexamethasone reduces TB antigen-induced IFN- $\gamma$ responses significantly using a QFT-GIT assay, without affecting responses of positive control [24]. There are variable results of QFT-GIT from patients who were on immunosuppressive therapies including steroids, oral immunosuppressant and biologic therapy. A negative effect of immunosuppressive therapy on the results of IGRA has also been shown in metaanalysis comprising of $71.5 \%$ of the patients who were on immunosuppressive treatment [25]. Use of corticosteroids and infliximab has shown to convert a positive QFT-GIT into negative of $30 \%$ of the study subjects tested [26]. Lowered lymphocyte counts are also found to be associated with indeterminate QFT-G results in patients with autoimmune disease [27]. Patients with lower $\mathrm{T}$ lymphocyte counts such immunocompromised or those with HIV infection have been shown to have false negative QFT-GIT results [28]. This is important to consider in countries where both TB and HIV are prevalent and recognition of latent as well as active TB has implications for treatment and control.

For EPTB, IGRAs have been employed as adjunct testing for investigation of $\mathrm{TB}$ disease and are recommended as such by ECDC and WHO guidelines [5, 17]. In our study cohort, the QFT-GIT had a specificity of $62 \%$ with a sensitivity of $76 \%$ for diagnosis of TB. In a metaanalysis of EPTB including disease at multiple sites by Fan et al., QFT-GIT was found to have a $72 \%$ specificity and $82 \%$ sensitivity for EPTB diagnosis [15]. In a metaanalysis of only tuberculosis lymphadenitis cases, QFTGIT specificity was $81 \%$ with a sensitivity of $89 \%$ for EPTB diagnosis [29]. The QFT-GIT sensitivity and specificity we found was lower than published studies possibly because the study design was one of consecutive convenient sampling. We did not select the study subjects and standardize the inclusion criteria. Instead we included cases referred by physicians for IGRA testing. It is possible that some of the study subjects were not suitable candidates for IGRA testing and this may have reduced the sensitivity and specificity of our study.

The AKUH hospital data illustrates that of the TB patients treated, $65 \%$ of the cases had EPTB (unpublished data). Diagnosis of MTB infection in patients with EPTB 
in a low resource setting remains problematic due to the inaccessibility of appropriate samples for testing and also due to financial considerations. As this was a retrospective study, one limitation is that we do not know how the IGRA results were utilized in management of the patient. However, from clinical records it was apparent that some patients with a negative IGRA but who had confirmation by other methods (culture, radiological) were put on anti-tuberculous treatment and were found to be responsive to it. Given our data, it appears that QFT-GIT IGRA is being used in our setting to identify MTB infection in clinically symptomatic individuals. It is only appropriate as an adjunct test if all other clinical parameters regarding the patient, other supporting laboratory and radiological test results are also taken into consideration.

\section{Conclusion}

As $50 \%$ of our study subjects had active TB, our study indicates that the QFT-GIT is being used as an adjunct test for diagnosis of TB in this setting. In our cohort, the specificity of QFT-GIT for diagnosis of TB was 62\% with a sensitivity of $76 \%$. The largest proportion of active TB cases had extrapulmonary TB. Given the variable impact of host immunity on IGRA test results, interpretation of QFT-GIT data for clinically symptomatic cases should be done with care, in the context of a full investigation of clinical, radiological parameters.

\section{Acknowledgements}

We thank Department of Pathology and Laboratory Medicine, the Aga Khan University for providing administrative support.

\section{Ethical approval}

The study was approved by Ethical Review Committee of the Aga Khan University, Karachi Pakistan. This was a retrospective review of hospital records of anonymized cases.

\section{Authors' contributions}

Conception and design of the study, or analysis and interpretation of data: $\mathrm{ZH}, \mathrm{KIM}, \mathrm{AA}, \mathrm{MI}, \mathrm{BJ}, \mathrm{MH}$. Manuscript write up: KIM, ZH, BJ. The author(s) read and approved the final manuscript.

\section{Funding}

This study did not receive any grant funding.

\section{Availability of data and material}

Upon request

\section{Consent for publication}

Not applicable.

\section{Competing interests}

The authors declare that they have no competing interests.

\section{Author details}

'Department of Pathology and Laboratory Medicine, The Aga Khan University, Stadium Road, P.O.Box 3500, Karachi, Pakistan. ${ }^{2}$ Department of Medicine, The Aga Khan University, Karachi, Pakistan.
Received: 9 July 2019 Accepted: 10 February 2020

Published online: 19 February 2020

\section{References}

1. WHO. Global Tuberculosis Report, vol. 2018. Geneva: World Health Organization; 2017.

2. WHO. Implementing the End TB strategy. Geneva: World Health Organization; 2015.

3. WHO. The End TB Strategy. In: Organization WH. 19th ed. Geneva: World Health Organization; 2015. WHO/HTM/TB/2015.

4. Diagnostic Standards and Classification of Tuberculosis in Adults and Children. Council of the Infectious Disease Society of America, September 1999. Am J Respir Crit Care Med. 2000;161 (4 Pt 1):1376-95.

5. WHO. Guidelines on the management of latent tuberculosis infection. Geneva: World Health Organization; 2015.

6. Pai M, Riley LW, Colford JM Jr. Interferon-gamma assays in the immunodiagnosis of tuberculosis: a systematic review. Lancet Infect Dis. 2004;4(12):761-76

7. Du F, Xie L, Zhang Y, Gao F, Zhang H, Chen W, et al. Prospective Comparison of QFT-GIT and T-SPOT.TB Assays for Diagnosis of Active Tuberculosis. Sci Rep. 2018;8(1):5882.

8. Centers for Disease Control and Prevention, Prevention NCFHVHSaT. TB Elimination: Tuberculin Skin Testing. 2011.

9. Muñoz L, Santin M, Alcaide F, Ruíz-Serrano MJ, Gijón P, Bermúdez E, et al. QuantiFERON-TB gold in-tube as a confirmatory test for tuberculin skin test in tuberculosis contact tracing: a noninferiority clinical trial. Clin Infect Dis. 2017:66:396-403.

10. Trehan I, O'Hare BA, Phiri A, Heikens GT. Challenges in the management of HIV-infected malnourished children in sub-Saharan Africa. AIDS Res Treat. 2012;2012:790786

11. Kordy F, Waters V, Den-Hollander C, Read S, Lam R, Kitai I. Optimizing blood collection and processing for Quantiferon-TB gold in-tube testing gives low rates of indeterminate results: clinical implications. Pediatr Infect Dis J. 2018; 37(1):e22-e4.

12. Trajman A, Steffen RE, Menzies D. Interferon-gamma release assays versus tuberculin skin testing for the diagnosis of latent tuberculosis infection: an overview of the evidence. Pulm Med. 2013;2013:601737.

13. Metcalfe JZ, Everett CK, Steingart KR, Cattamanchi A, Huang L, Hopewell PC, et al. Interferon-gamma release assays for active pulmonary tuberculosis diagnosis in adults in low- and middle-income countries: systematic review and meta-analysis. J Infect Dis. 2011:204(Suppl 4):S1120-9.

14. Dai Y, Feng Y, Xu R, Xu W, Lu W, Wang J. Evaluation of interferon-gamma release assays for the diagnosis of tuberculosis: an updated meta-analysis. Eur J Clin Microbiol Infect Dis. 2012;31(11):3127-37.

15. Fan $\mathrm{L}$, Chen $\mathrm{Z}$, Hao XH, Hu ZY, Xiao HP. Interferon-gamma release assays for the diagnosis of extrapulmonary tuberculosis: a systematic review and meta-analysis. FEMS Immunol Med Microbiol. 2012;65(3):456-66.

16. Lavender TW, Barrett A, Magee J, Ong EL. Interferon-gamma release assays in the diagnosis of active tuberculosis disease in a low-incident setting: a 5year review of data. Clin Microbiol Infect. 2013;19(11):1078-81.

17. European Centre for Disease Prevention and Control. Use of interferongamma release assays in support of TB diagnosis. Stockholm: ECDC; 2011. Stockholm, March 2011 ISBN 978-92-9193-240-5. https://doi.org/10.2900/ 38588.

18. Jeon CY, Murray MB. Diabetes mellitus increases the risk of active tuberculosis: a systematic review of 13 observational studies. PLoS Med. 2008:5(7):e152.

19. Stevenson $C R$, Forouhi $N G$, Roglic $G$, Williams $B G$, Lauer JA, Dye C, et al. Diabetes and tuberculosis: the impact of the diabetes epidemic on tuberculosis incidence. BMC Public Health. 2007;7:234.

20. Mensah Gl, Sowah SA, Yeboah NY, Addo KK, Jackson-Sillah D. Utility of QuantiFERON tuberculosis gold-in-tube test for detecting latent tuberculosis infection among close household contacts of confirmed tuberculosis patients in Accra. Ghana Int J Mycobacteriol. 2017;6(1):27-33.

21. Qiu X, Tang Y, Zou R, Zeng Y, Yue Y, Li W, et al. Diagnostic accuracy of interferon-gamma-induced protein 10 for differentiating active tuberculosis from latent tuberculosis: a meta-analysis. Sci Rep. 2019:9(1):11408.

22. Munoz L, Santin M. Interferon-gamma release assays versus tuberculin skin test for targeting people for tuberculosis preventive treatment: an evidencebased review. J Inf Secur. 2013;66(4):381-7. 
23. Theel ES, Hilgart H, Breen-Lyles M, McCoy K, Flury R, Breeher LE, et al. Comparison of the QuantiFERON-TB Gold Plus and QuantiFERON-TB Gold In-Tube Interferon Gamma Release Assays in Patients at Risk for Tuberculosis and in Health Care Workers. J Clin Microbiol. 2018:56(7):e00614-18.

24. Clifford V, Zufferey C, Germano S, Ryan N, Leslie D, Street A, et al. The impact of anti-tuberculous antibiotics and corticosteroids on cytokine production in QuantiFERON-TB gold in tube assays. Tuberculosis (Edinb). 2015;95(3):343-9.

25. Wong SH, Gao Q, Tsoi KK, Wu WK, Tam LS, Lee N, et al. Effect of immunosuppressive therapy on interferon gamma release assay for latent tuberculosis screening in patients with autoimmune diseases: a systematic review and meta-analysis. Thorax. 2016;71(1):64-72.

26. Edwards A, Gao Y, Allan RN, Ball D, de Graaf H, Coelho T, et al. Corticosteroids and infliximab impair the performance of interferongamma release assays used for diagnosis of latent tuberculosis. Thorax. 2017;72(10):946-9.

27. Gonzalez-Moreno J, Garcia-Gasalla M, Losada-Lopez I, Cifuentes Luna C, Mi Viladrich I, Fernandez-Baca V, et al. IGRA testing in patients with immunemediated inflammatory diseases: which factors influence the results? Rheumatol Int. 2018:38(2):267-73.

28. Takenami I, Loureiro C, Machado A Jr, Emodi K, Riley LW, Arruda S. Blood cells and interferon-gamma levels correlation in latent tuberculosis infection. ISRN Pulmonol. 2013;2013. https://doi.org/10.1155/2013/256148

29. Liu Q, Li W, Chen Y, Du X, Wang C, Liang B, et al. Performance of interferongamma release assay in the diagnosis of tuberculous lymphadenitis: a meta-analysis. PeerJ. 2017:5:e3136.

\section{Publisher's Note}

Springer Nature remains neutral with regard to jurisdictional claims in published maps and institutional affiliations.

Ready to submit your research? Choose BMC and benefit from:

- fast, convenient online submission

- thorough peer review by experienced researchers in your field

- rapid publication on acceptance

- support for research data, including large and complex data types

- gold Open Access which fosters wider collaboration and increased citations

- maximum visibility for your research: over $100 \mathrm{M}$ website views per year

At BMC, research is always in progress.

Learn more biomedcentral.com/submissions 